\title{
TEM analysis as a tool for toxicological assessment of occupational exposure to airborne nanoparticles from welding
}

\author{
J. Gomes***, C. Guerreiro***, D. Lavrador***, P.A. Carvalho**** and R.M. Miranda*** \\ *IBB - Instituto de Biotecnologia e Bioengenharia, Instituto Superior Técnico, Universidade Técnica de Lisboa, \\ Av. Rovisco Pais, 1049-001, Lisboa, Portugal \\ **ISEL - Instituto Superior de Engenharia de Lisboa, R. Conselheiro Emídio Navarro, 1959-007, Lisboa, \\ Portugal \\ ***UNIDEMI - Departamento de Engenharia Mecânica e Industrial, Faculdade de Ciências e Tecnologia, \\ Universidade Nova de Lisboa, 2829-516, Caparica, Portugal \\ **** ICEMS - Instituto Superior Técnico, Universidade de Lisboa, Av. Rovisco Pais, 1049-001, Lisboa, Portugal
}

Welding is the principal industrial process used for joining metals. However, it can produce dangerous fumes that may be hazardous to the welder's health and it is estimated that, presently, $1-2 \%$ of workers from different professional backgrounds (which accounts for more than 3 million persons) are subjected to welding fume and gas action. With the advent of new types of welding procedures and consumables, the number of welders exposed to welding fumes is growing constantly in spite of the mechanization and automation of the processes. Simultaneously, the number of publications on epidemiologic studies and the devices for welders' protection is also increasing. Apart from that, the influence of very ultrafine particulate, lying in the nanoparticles range, on human health has been pointed to be of much concern as airborne nanoparticles are resulting both from nanotechnologies processes and also from macroscopic common industrial processes such as welding. In fact, nanotoxicological research is still in its infancy and the issuing and implementation of standards for appropriate safety control systems can still take several years. Yet, the advanced understanding of toxicological phenomena on the nanometre scale is largely dependent on technological innovations and scientific results stemming from enhanced R\&D. Meanwhile, the industry has to adopt proactive risk management strategies in order to provide a safe working environment for their staff, clients and customers, and obtain products without posing health threats at any point of their lifecycle. Understanding the relationship of airborne nano sized particulate and human health, under different environmental conditions is of great importance for improving exposure estimates and for developing efficient control strategies to reduce human exposure and health risk and for establishing, evaluating and improving regulations and legislation both on air quality, airborne emissions and the incorporation of nano sized materials in other products and commodities [1].

Mass measurement methods are not sufficiently sensitive for airborne nanoparticles and are not sensitive toward the specific health relevant properties of nanoparticles [2,3]. The most sensitive concentration measured in this particle range $(<100 \mathrm{~nm}$ diameter) is the number concentration. In fact, surface area is a relevant metric for nanoparticles, as most of the processes in the human body environment take place via the particle surface, which is increasing significantly with decreasing particle size in the nanometer size range for the same amount of mass. In order to perform the toxicological assessment of welding processes a Nanoparticle Surface Area Monitor, TSI, Model 3550, based on diffusion charging (measuring the electrostatic charge on a sampled aerosol, mainly composed of nanoparticles) was used for monitoring the emission of nanoparticles resulting from several welding processes. This equipment indicates the human lung-deposited surface area of particles expressed as square micrometers per cubic centimeter of air $\left(\mu \mathrm{m}^{2} / \mathrm{cm}^{3}\right)$ [4]. Although this instrument is very precise and its use has been validated for this purpose, the definite presence of nanoparticles in welding fume has to be complemented by microscopy techniques such as transmission electron microscopy (TEM), which has proved very helpful in order to establish the size, shape and aggregation habit of sampled aerosols, as well as energy dispersive X-ray spectroscopy (EDS) for performing the chemical analysis of collected nanoparticles. Figure 1 shows the aspect of aggregates of particles, in the nano size range, collected from welding fume during shielded metal arc welding (SMAW) of carbon steel and friction stir welding (FSW) of aluminium alloys, respectively. As expected in arc welding aggregates of larger dimensions are produced in more roundish shaped particles while in FSW these are finer. Chemical analysis determined by EDS showed that the emitted nanoparticles of SMAW are 
mostly resulting from burning of electrode coating and less from its core. Therefore, in order to reduce particulate emissions, special care has to be paid to the chemical composition of the electrodes coating.

\section{References}

1. Gomes J. et al., Tox. and Env. Health A, 75:1-9, 2012.

2. Pires I. et al., Tox. \& Env. Chem., 88:385-394, 2006.

3. Pires I. et al., Mat. and Des., 28:1623-1631, 2007.

4. Gomes J.F. et al., Inh. Tox., 24:425-433, 2012.
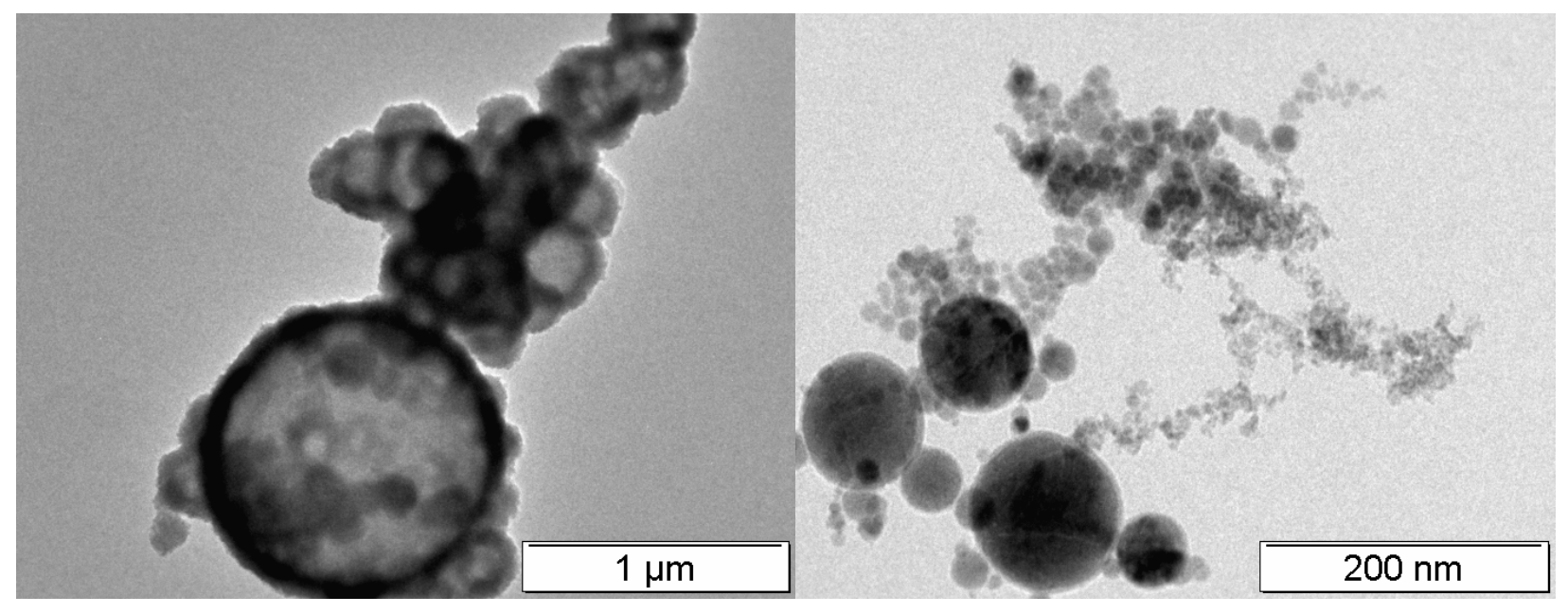

Figure 1. TEM images of aerosols in welding fumes: Left: SMAW of carbon steel with basic coated electrodes. Right: from friction stir welding of aluminium alloys.

The work was supported by the Portuguese Science Foundation through PEst-OE/EQB/LA0023/2011, PEstOE/EME/UI0667/2011 and PEst-OE/CTM-UI0084/2011 and grants. 